\title{
Minireview
}

\section{Targeting cytotoxic $\top$ lymphocytes for cancer immunotherapy}

\author{
J Maher*,1,2 and ET Davies ${ }^{2}$ \\ 'Cancer Research UK Breast Cancer Biology Group, Division of Cancer Studies, Guy's King's and St Thomas's School of Medicine, Guy's Hospital, St \\ Thomas Street, London SEI 9RT, UK; '2Department of Clinical Immunology, King's College Hospital, The Rayne Institute, I 23 Coldharbour Lane, London \\ SE5 9NU, UK
}

In light of their preeminent role in cellular immunity, there is considerable interest in targeting of cytotoxic T-lymphocytes to cancer. This review summarises the active and passive immunotherapeutic approaches under development to achieve this goal, emphasising how recent advances in tumour immunology and gene transfer have impacted upon this field. British Journal of Cancer (2004) 91, 817-821. doi:10.1038/sj.bjc.6602022 www.bjcancer.com Published online 13 July 2004

(c) 2004 Cancer Research UK

Keywords: CTL; vaccine; adoptive immunotherapy; gene therapy

The challenge of developing cancer immunotherapy is one of the longest standing goals of immunology, dating back to the late 19th century. Despite many setbacks, recent developments have rejuvenated the sense of optimism in this quest. Almost 30 years after their development, monoclonal antibodies are now commonly used in the treatment of selected malignancies. While this represents an important advance, much evidence suggests that harnessing of the cellular immune system might contribute even more effectively to this endeavour. The focus of this minireview is to consider how $\mathrm{CD}^{+}$cytotoxic $\mathrm{T}$ lymphocytes (CTL) might be targeted for cancer immunotherapy.

\section{CYTOTOXIC T LYMPHOCYTES AS EFFECTORS OF ANTITUMOUR IMMUNITY}

Cytotoxic $\mathrm{T}$ lymphocytes represent a crucial component of the adaptive immune system with particular importance in the control of intracellular pathogens. Effector CTL have the capacity to promote the apoptotic death of carefully chosen target cells, using a combination of granule (perforin/granzyme)and receptor (Fas/tumour necrosis factor)-mediated mechanisms. While natural killer cells also promote cell death, CTL are distinguished by their exquisite specificity for antigen, which they recognise using a clonally unique $\mathrm{T}$-cell receptor (TCR). Target cells are 'flagged' for the attention of CTL when they present antigen-derived peptide fragments on the cell surface, inserted into the groove of class I major histocompatibility (MHC) molecules.

In addition to cytolytic function, a number of other properties render CTL attractive as mediators of antitumour immunity. First, the widespread expression of MHC class I molecules means that

\footnotetext{
* Correspondence: Dr J Maher, Cancer Research UK Breast Cancer Biology Group, Division of Cancer Studies, Guy's King's and St Thomas's School of Medicine, Guy's Hospital, St Thomas Street, London SEI 9RT, UK; E-mail: john.maher@cancer.org.uk

Received 12 February 2004; revised 21 May 2004; accepted 26 May 2004; published online 13 July 2004
}

CTL could, in principle, be deployed against malignancies of diverse origin. Second, CTL (like all lymphocytes) continuously recirculate throughout the body to seek out antigen, a useful property for the treatment of systemic disease. Third, target recognition is impressively sensitive - even a single peptide-MHC class I complex may trigger cytolysis by a high-avidity effector CTL (Sykulev et al, 1996). Finally, CTL also employ nonlytic effector mechanisms including the production of interferon gamma - a cytokine with several direct and indirect antitumour properties (Qin et al, 2003).

In light of their lethal payload, generation of effector CTL is tightly regulated and depends upon the antigen-driven differentiation of naïve $\mathrm{CD} 8{ }^{+} \mathrm{T}$ cells. This process is initiated when dendritic cells (DC) take up antigen within peripheral tissues and migrate to the regional lymph nodes. Here, DC are ideally placed to interact with naïve $\mathrm{T}$ lymphocytes, which preferentially recirculate through secondary lymphoid tissue. In order to achieve optimum CTL priming, DC must be in an activated ('licensed') state. This is generally achieved when DC present processed antigen to MHC class II-restricted $\mathrm{CD}^{+}$(helper) $\mathrm{T}$ cells - the latter then upregulate CD40 ligand that engages DC-associated CD40. Alternatively, DC may be licensed in response to some proinflammatory cytokines or bacterial products that engage Toll-like receptors (Lanzavecchia, 1998).

To achieve optimum expansion of CTL, two additional requirements should be met. First, since $\mathrm{CD} 4^{+}$and $\mathrm{CD}{ }^{+} \mathrm{T}$ cells are restricted by different MHC families, it is desirable that DC process antigen to enable presentation of derived epitopes both by MHC class I and II. Second, appropriate costimulatory signals must be provided ('signal 2') to complement that delivered by the TCR ('signal 1'). These accessory signals are delivered by several ligands, many of which are upregulated on licensed DC. The best characterised of these are the members of the B7 family, which engage T-cell-associated CD28. Under some circumstances, the resultant clonal expansion of CTL is quite remarkable. In the context of some acute viral infections, CTL of a single specificity may account for more than $40 \%$ of peripheral blood CD8 ${ }^{+} \mathrm{T}$ cells (Callan et al, 1998). 


\section{IMMUNE TOLERANCE AND CANCER}

To counteract the attention of CTL and other effector arms of the immune system, cancer cells deploy several immune evasion strategies (summarised in Figure 1). Many of these diversionary tactics rely upon the ability of cancer cells to harness systems that maintain immune tolerance to self. Immune tolerance is mediated in part by deletion of autoreactive lymphocytes, although this would be difficult to reverse using an immunotherapeutic approach. By contrast, nondeletional tolerance mechanisms may in principle prove more amenable to therapeutic manipulation. For example, increasing evidence has implicated a number of regulatory $\mathrm{T}$-cell subsets in the maintenance of immune tolerance both to self and to derived malignancies. These include CD4 ${ }^{+}$ CD25 ${ }^{+}$Treg cells, IL-10-producing Tr1 cells, transforming growth factor (TGF)- $\beta$-producing Th3 cells and $\mathrm{CD} 8{ }^{+}$regulatory $\mathrm{T}$-cell subsets (Nelson, 2004). In light of this, it is conceivable that the depletion or suppression of such cellular populations might provide a useful adjunct in the immunotherapy of malignant disease.

\section{VACCINATION STRATEGIES THAT TARGET CTL TO TUMOUR ANTIGENS}

In principle, CTL may be targeted to cancer using one of two approaches, based upon the classical establishment of active or passive immunity. The greatest success of clinical immunology has been the development of vaccines for the prophylaxis of infectious disease. Indeed, some such vaccines are proving useful in the prevention of virus-related malignant disorders, notably hepatoma

Tumour cell

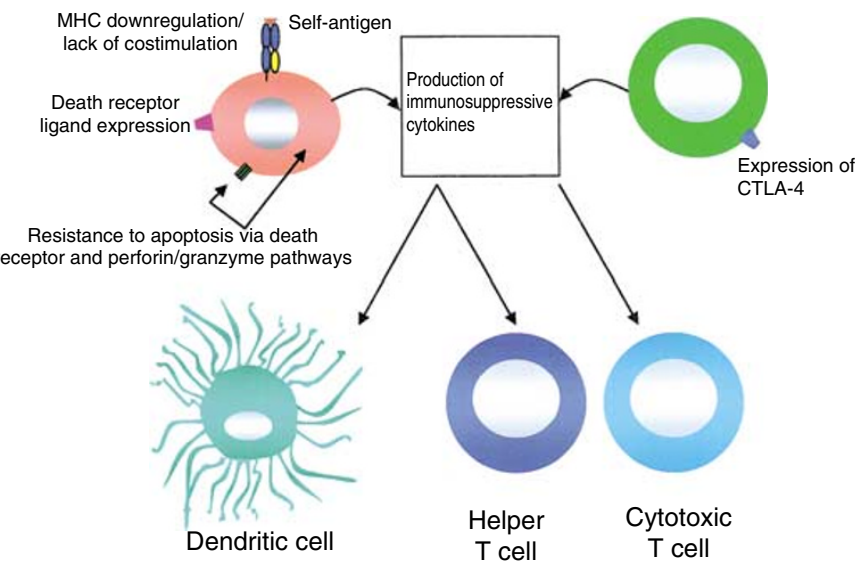

Figure I Tumours can escape from immune targeting by CTL in several ways. First, since cancer cells are closely related to 'self', they contain a predominance of poorly immunogenic self-antigen. Second, cancer cells have poor antigen-presenting properties since they provide limited costimulation and may downregulate $\mathrm{MHC}$ antigen expression. Third, the tumour microenvironment is frequently rich in cytokines that compromise DC differentiation (e.g. interleukin (IL)-6, IL-I0, macrophage colonystimulating factor, vascular endothelial growth factor) or activation, proliferation and effector function of $T$ lymphocytes (e.g. transforming growth factor- $\beta$ ). Fourth, cancer cells may acquire resistance to apoptosis, a key effector mechanism of CTL and other cells of the immune system. Of related significance, cancer cells may express death receptor ligands (e.g. Fas ligand) that could facilitate the elimination of tumour-specific $T$ cells ('immune counterattack'). Finally, it has been suggested that regulatory $T$ cell subsets (for example, CD4 ${ }^{+} \mathrm{CD}_{2} 5^{+}$Treg cells) promote immune tolerance to cancer. These cells also produce immunosuppressive cytokines and express CTLA-4, a molecule with a number of tolerance-promoting activities.
(Chang et al, 1997). The majority of infectious disease vaccines achieve host immunity since they elicit a protective antibody response. By contrast, most tumour vaccines have focused upon the induction of tumour-reactive CTL.

One traditional approach to the development of a cancer vaccine involves the use of inactivated whole tumour cells (or derived extracts - Figure 2A). More recently, this approach has been extended with the development of tumour cell-based vaccines engineered to express immunomodulatory cytokines and/or costimulatory ligands (e.g. following genetic modification or fusion with DC). An important difficulty with the use of such complex vaccines is the fact that relevant antigens are often obscure and thus in vitro immune monitoring may prove difficult.

In recent years, the scientific rationale underlying tumour vaccination has been strengthened enormously by the demonstration that nonviral malignancies express tumour antigens. This crucial advance, pioneered by Thierry Boon and co-workers (van der Bruggen et al, 2002), has resulted in the development of vaccines based upon defined molecular targets. In the majority of cases, such antigens are targets for CTL and derive from molecules
A

Tumour cells and derivatives

0000

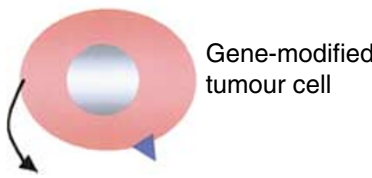

GM-CSF Costimulation

C

Viral vaccine

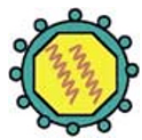

B

Tumour antigen-specific vaccines

Tumour cell debris, exosomes, etc.

\section{MOMAMA DNA}

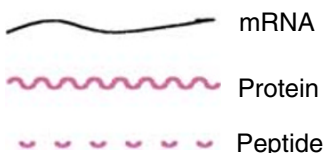

D

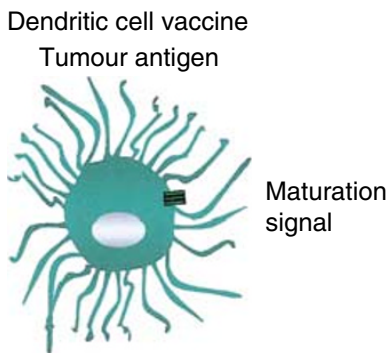

Figure 2 To break immune tolerance against cancer, several vaccinebased strategies are under development. (A) To target a broad spectrum of antigens, tumour cells may be engineered to secrete immunomodulatory cytokines, such as GM-CSF, and/or costimulatory ligands (e.g. B7) Alternatively, whole tumour cell derivatives may be administered together with an appropriate adjuvant and/or delivery system. (B) Vaccines based upon discrete tumour antigen(s) may be formulated in several ways - for example, as naked DNA, protein or derived peptide(s). Since many tumour antigen-derived peptides bind weakly to $\mathrm{MHC}$, sequence alterations may be incorporated to increase binding affinity (heteroclitic approach). A variety of targeting motifs may be fused with such molecular vaccines in an attempt to direct delivery (for example to dendritic cells) and/or preferential processing by the MHC class I or class II pathway. (C) Viral delivery systems are powerfully immunogenic, particularly when live (attenuated) vectors are used. These agents show particular promise in 'prime-boost' strategies, in which antigen is administered sequentially by distinct means. (D) Dendritic cells are potent antigen-presenting cells that may be used to deliver tumour antigen in several forms. Whereas immature DC may promote tolerance, mature DC are highly immunogenic and may even break tolerance to self-antigen. 
that are overexpressed in tumour cells, compared to their normal counterparts. The recognition of the close relationship between tumour antigens and 'self' provides a molecular explanation for one of the key obstacles to the breaking of immune tolerance to cancer. In light of this difficulty, a very large number of antigenspecific vaccines are under development (Figure 2B-C), based upon peptides, protein, mRNA or DNA expressed from plasmid or viral vectors (Whelan et al, 2003). To enhance immunogenicity, a range of adjuvants have been tested many of which promote DC activation. Indeed, an increasing area of study involves the use of DC-based vaccines engineered to express antigen (Figure 2D; Cerundolo et al, 2004). Many tumour vaccines have successfully expanded CTL responses to appropriate antigens (Coulie and van der Bruggen, 2003). Furthermore, some promising studies have been reported in experimental animal models, particularly in the setting of protection from tumour challenge (Morris et al, 2003).

Recently, several small clinical studies of therapeutic vaccination have been performed. In general, these trials have confirmed the safety of the approach and have sometimes demonstrated induction of CTL and/or antibody responses to vaccine components (e.g. Knutson et al, 2001). These findings suggest that tumours bearing defined antigens can be targeted immunotherapeutically. Somewhat disappointingly however, apart from sporadic responses in isolated individuals, clinical outcomes have generally been disappointing (Finn, 2003; Morris et al, 2003).

What lessons have been learned that might improve the effectiveness of tumour vaccination? A key point illustrated by many animal studies is the relative ineffectiveness of this approach in the setting of advanced tumour burden. Consequently, it may prove much more fruitful to test suitable vaccines in patients with minimal residual disease. A further option is to combine vaccination with other therapeutic modalities, as recently illustrated in acute promyelocytic leukaemia (Padua et al, 2003). Third, it may be worth developing broadly applicable vaccines that simultaneously target CTL to multiple tumour-associated epitopes (Graff-Dubois et al, 2002). In parallel, it is important to determine why therapeutic vaccination is poorly effective in advanced disease and to understand those instances where significant responses are observed. A variety of new techniques permit the monitoring of antigen-specific CTL responses, including ELISPOT, tetramer analysis and intracellular cytokine detection (Clay et al, 2001). While studies to date have only occasionally correlated results of such assays with clinical responses, this is likely to provide a fertile area for further research.

\section{ADOPTIVE IMMUNOTHERAPY USING EX VIVO GEN- ERATED CTL}

Passive (adoptive) immunotherapeutic approaches also represent an attractive means to target CTL to tumour cells. In some in vivo experimental models, adoptive immunotherapy using amplified CTL has demonstrated greater success than vaccination approaches that target the same tumour type (Romieu et al, 1998). Adoptive immunotherapy generally involves the administration of large numbers of $\mathrm{T}$ cells, thereby bypassing tolerance mechanisms that limit the activation and expansion of CTL. In clinical practice, this approach is best illustrated by treatment of some haematological malignancies with allogeneic stem cell transfer, or donor leucocyte infusion. In this setting, there is compelling evidence that CTL and other effector cells deliver a 'graft $v s$ leukaemia effect' that contributes importantly to therapeutic efficacy. Transferred CTL are also of established benefit in the treatment of some virusrelated malignancies, such as Epstein-Barr virus (EBV)-related post-transplant lymphoproliferative disease (Rooney et al, 2001). However, evidence that such cellular therapies can achieve meaningful control of common solid tumours is more limited. Furthermore, it is well known that allogeneic T cells can mediate graft $v s$ host disease, with potentially lethal consequences. Consequently, there is a need to broaden the applicability and enhance the safety of this approach, preferably with the use of tumour-specific autologous T lymphocytes.

The development of adoptive T-cell immunotherapy for solid tumours has been pioneered by Steven Rosenberg and co-workers. Following the demonstration that IL-2 could achieve responses in a small number of patients with malignant melanoma, this group subsequently developed techniques to expand tumour-infiltrating lymphocytes (TIL) in vitro. When TIL were infused into patients, a modest improvement in response rate became apparent, although success was hampered by poor in vivo persistence of transferred cells (Rosenberg et al, 1994). Over the ensuing years, it has emerged that TIL are enriched for MHC class I-restricted CTL with specificity for known melanoma antigens including MART-1 and gp100. More recently, this group have shown that following lymphodepletion, TIL undergo impressive IL-2-driven expansion in vivo, increasing the clinical response rate significantly (Dudley et al, 2002).

These studies elegantly provide proof of principle for the clinical potential of adoptive immunotherapy. To translate this approach more widely, systems are required to achieve the rapid ex vivo expansion of CTL targeted to relevant tumour antigens. Fortunately, a number of strategies are under development that may achieve this.

\section{GENETIC APPROACHES TO CTL-BASED ADOPTIVE IMMUNOTHERAPY}

A recent key development has been the application of gene transfer-based strategies to target CTL to cancer cells. Several avenues of investigation are under development at this time. One promising approach involves the use of 'artificial antigenpresenting cells' that permit the in vitro expansion of tumourspecific CTL (Latouche and Sadelain, 2000). To achieve this, NIH3T3 fibroblasts have been genetically engineered to express a chosen peptide epitope, together with an MHC class I molecule, $\beta 2$ microglobulin and a series of costimulatory ligands. Using this system, impressive expansion of CTL of the desired specificity can be achieved. To further boost in vitro CTL expansion, many groups have demonstrated the potent growth-promoting effect of IL-15 (e.g. Brentjens et al, 2003).

An alternative approach is to genetically modify T cells or CTL, thereby retargeting specificity to a chosen tumour antigen (Eshhar, 1997). Most commonly, this is achieved using a 'chimeric antigen receptor' (CAR) in which an antibody-derived single chain fragment $(\mathrm{scFv})$ is coupled via a hinge to an appropriate signalling element (Figure 3). Chimeric antigen receptors are generally expressed on the $\mathrm{T}$-cell surface as a single gene-encoded homodimer, enabling the MHC-independent recognition of native tumour-associated antigen. Several such fusion receptors have been constructed with specificity for molecules expressed by solid and haematological malignancies (e.g. Eshhar, 1997, Gong et al, 1999).

In most early CAR designs, the signalling domain was chosen to deliver a surrogate TCR-like stimulus. The CD3 $\zeta$ cytoplasmic domain delivers a potent signal 1 and is well suited for this purpose. Despite fears about TCR signalling in cancerbearing hosts, it is reassuring that $\mathrm{CD} 3 \zeta$-based $\mathrm{CAR}$ retain potent activity when expressed in T lymphocytes derived from such patients (Gong et al, 1999; Brentjens et al, 2003; Sheen et al, 2003).

Most tumours do not provide adequate T-cell costimulation. Consequently, there has been considerable interest in the development of CAR that provides such accessory signals. An 


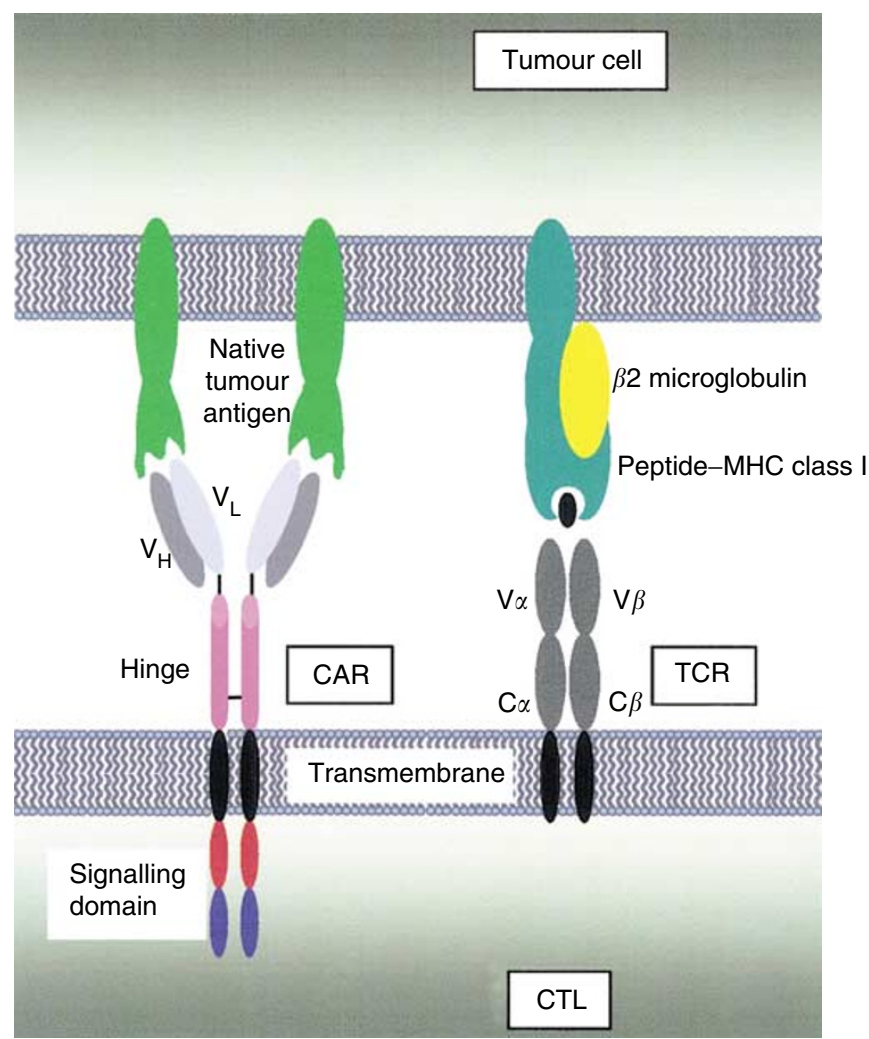

Figure 3 Antigen recognition by chimeric antigen receptors (CAR) is most commonly mediated by a single chain antibody fragment, comprising a variable heavy $(\mathrm{VH})$ and variable light $(\mathrm{VL})$ chain domain, joined with a short linker (left). In recent CAR designs, the intracellular domain comprises a modular array of fused signalling elements that together provide a TCRlike and a costimulatory signal. By contrast, the TCR recognises processed antigen, presented as a peptide $-\mathrm{MHC}$ complex (right). This in turn triggers signal transduction by the CD3 complex (not shown).

important initial development was the construction of scFv/CD28 fusion receptors (Alvarez-Vallina and Hawkins, 1996). More recently, CAR have been designed in which the signalling domains of $\mathrm{CD} 3 \zeta$ and $\mathrm{CD} 28$ have been fused in series, creating molecules that can deliver both a functional signal 1 and signal 2 (Finney et al, 1998). When such 'second-generation' CAR are expressed in human $\mathrm{T}$ cells, they can be repeatedly activated in vitro by coculture with antigen-expressing tumour cells. Each cycle of stimulation results in rapid death of the tumour targets, followed by IL-2-driven proliferation of CAR-grafted T cells (Maher et al, 2002). More recently, it has been shown that several alternative costimulatory domains can be fused in series with $\mathrm{CD} 3 \zeta$ to create CAR with distinct functional properties (Finney et al, 2004). An alternative approach to promote sustained survival of CAR-grafted CTL involves selective gene-transfer into EBV-responsive T cells, which are known to persist for prolonged periods in vivo (Rossig et al, 2002). In parallel to these developments, CAR technology has received an important boost with the demonstration that this approach can achieve sustained control of an established in vivo tumour burden (Haynes et al, 2002; Brentjens et al, 2003). Significantly, those studies have also emphasised the importance of costimulation.

An alternative genetic strategy involves the introduction of a new TCR with specificity for a defined, tumour-associated peptide-MHC complex. A number of in vitro studies have successfully used this system to redirect the antigenic specificity of both $\mathrm{CD}^{+}$and $\mathrm{CD} 8{ }^{+} \mathrm{T}$ cells. This approach is attractive in that it allows access to a greater repertoire of protein antigens than CAR, since the latter requires that the targeted antigen is expressed on the cell surface. Furthermore, it might be anticipated that ectopic TCR would prove less immunogenic than CAR that originate from (nonhumanised) rodent hybridoma-based scFv. However, these advantages are balanced by some important disadvantages. Since the TCR is a heterodimer, this strategy requires the regulated coexpression of two gene products (TCR $\alpha$ and $\beta$ chains). In principle, such exogenous receptor subunits may heterodimerise with endogenous TCR subunits, generating complexes with autoreactive potential. This potential difficulty may be overcome by inclusion of sequences that only permit dimerisation of the ectopic TCR subunits. A second disadvantage is the frequent downregulation of MHC class I molecules observed in cancer, providing an opportunity for immune escape from the genetically modified CTL (Gilboa, 1999). Thirdly, owing to the highly polymorphic nature of the human MHC (human leucocyte antigen - HLA) system, ectopic TCR would need to be matched to the HLA haplotype of the patient, imposing additional logistical constraints.

An important consideration for all forms of immunotherapy is the tumour microenvironment, which is frequently poorly conducive to CTL function (Figure 1). Many malignancies are associated with overproduction of immunosuppressive cytokines such as TGF- $\beta$, although genetic approaches may be used to circumvent this (Gorelik and Flavell, 2001). Alternatively, the microenvironment may be decorated with a suitable immunopotentiating agent, such as the tumour-necrosis factor superfamily member, LIGHT. Recently, this has been reported to achieve a dramatic recruitment of naïve CTL to malignant deposits, where they are primed and elicit impressive antitumour immunity ( $\mathrm{Yu}$ et al, 2004).

The exciting opportunities afforded by genetic modification of $\mathrm{T}$ cells have set the stage for a number of clinical studies that are currently underway. However, a cautionary note is warranted in light of the development of leukaemia in two children following treatment of X-linked severe combined immunodeficiency using gene-modified haemopoietic stem cells. A full discussion of this complex issue is beyond the scope of the current review (see Kohn et al, 2003). It is clear, however, that safety must be paramount in the clinical testing of such novel treatments. Consequently, it may be appropriate to include 'suicide genes' in adoptively transferred $\mathrm{T}$ cells to allow their elimination when required. The best studied of these approaches involves expression of the herpes simplex virus thymidine kinase gene, which renders $\mathrm{T}$ cells susceptible to ganciclovir.

\section{CONCLUSIONS}

Recent developments mean that immunotherapy is likely to play an increasingly important role in cancer therapy. Adoptive immunotherapy may find its niche in the debulking of disease that is resistant to conventional therapeutic regimens. By contrast, vaccination may prove most useful in the context of lower tumour burden and as a means to attain longer-term immunity and memory. In both settings, CTL are likely to play a key role, justifying the widespread interest in targeting of these key immune effector cells to cancer.

\section{ACKNOWLEDGEMENTS}

John Maher is supported by a Senior Clinician Scientist Research Fellowship (awarded by the Royal College of Pathologists and the Healthcare Foundation) and by the Breast Cancer Campaign. We gratefully acknowledge Mohammad Ibrahim for review of the manuscript. 


\section{REFERENCES}

Alvarez-Vallina L, Hawkins RE (1996) Antigen-specific targeting of CD28-mediated $\mathrm{T}$ cell co-stimulation using chimeric single-chain antibody variable fragment-CD28 receptors. Eur J Immunol 26: $2304-2309$

Brentjens RJ, Latouche JB, Santos E, Marti F, Gong MC, Lyddane C, King PD, Larson S, Weiss M, Riviere I, Sadelain M (2003) Eradication of systemic B-cell tumors by genetically targeted human T lymphocytes costimulated by CD80 and interleukin-15. Nat Med 9: 279-286, doi: $10.1038 / \mathrm{nm} 827$

Callan MF, Tan L, Annels N, Ogg GS, Wilson JD, O’Callaghan CA, Steven N, McMichael AJ, Rickinson AB (1998) Direct visualization of antigenspecific CD8+ T cells during the primary immune response to Epstein Barr virus in vivo. J Exp Med 187: $1395-1402$

Cerundolo V, Hermans IF, Salio M (2004) Dendritic cells: a journey from laboratory to clinic. Nat Immunol 5: 7 - 10, doi:10.1038/ni0104-7

Chang MH, Chen CJ, Lai MS, Hsu HM, Wu TC, Kong MS, Liang DC, Shau WY, Chen DS (1997) Universal hepatitis B vaccination in Taiwan and the incidence of hepatocellular carcinoma in children. Taiwan Childhood Hepatoma Study Group. N Engl J Med 336: 1855-1859

Clay TM, Hobeika AC, Mosca PJ, Lyerly HK, Morse MA (2001) Assays for monitoring cellular immune responses to active immunotherapy of cancer. Clin Cancer Res 7: 1127-1135

Coulie PG, van der Bruggen P (2003) T-cell responses of vaccinated cancer patients. Curr Opin Immunol 15: 131-137, doi:10.1016/S09527915(03)00009-8

Dudley ME, Wunderlich JR, Robbins PF, Yang JC, Hwu P, Schwartzentruber DJ, Topalian SL, Sherry R, Restifo NP, Hubicki AM, Robinson MR, Raffeld M, Duray P, Seipp CA, Rogers-Freezer L, Morton KE, Mavroukakis SA, White DE, Rosenberg SA (2002) Cancer regression and autoimmunity in patients after clonal repopulation with antitumor lymphocytes. Science 298: $850-854$

Eshhar Z (1997) Tumor-specific T-bodies: towards clinical application. Cancer Immunol Immunother 45: $131-136$

Finn OJ (2003) Cancer vaccines: between the idea and the reality. Nat Rev Immunol 3: $630-641$, doi:10.1038/nri1150

Finney HM, Akbar AN, Lawson AD (2004) Activation of resting human primary $\mathrm{T}$ cells with chimeric receptors: costimulation from $\mathrm{CD} 28$, inducible costimulator, CD134, and CD137 in series with signals from the TCR zeta chain. J Immunol 172: 104-113

Finney HM, Lawson AD, Bebbington CR, Weir AN (1998) Chimeric receptors providing both primary and costimulatory signaling in $\mathrm{T}$ cells from a single gene product. I Immunol 161: 2791-2797

Gilboa E (1999) How tumors escape immune destruction and what we can do about it. Cancer Immunol Immunother 48: 382-385

Gong MC, Latouche JB, Krause A, Heston WD, Bander NH, Sadelain M (1999) Cancer patient $\mathrm{T}$ cells genetically targeted to prostate-specific membrane antigen specifically lyse prostate cancer cells and release cytokines in response to prostate-specific membrane antigen. Neoplasia 1: $123-127$

Gorelik L, Flavell RA (2001) Immune-mediated eradication of tumors through the blockade of transforming growth factor-beta signaling in $\mathrm{T}$ cells. Nat Med 7: 1118-1122, doi:10.1038/nm1001-1118

Graff-Dubois S, Faure O, Gross DA, Alves P, Scardino A, Chouaib S, Lemonnier FA, Kosmatopoulos K (2002) Generation of CTL recognizing an HLA-A*0201-restricted epitope shared by MAGE-A1, -A2, -A3, -A4, A6, -A10, and -A12 tumor antigens: implication in a broad-spectrum tumor immunotherapy. J Immunol 169: 575-580

Haynes NM, Trapani JA, Teng MW, Jackson JT, Cerruti L, Jane SM, Kershaw MH, Smyth MJ, Darcy PK (2002) Single-chain antigen recognition receptors that costimulate potent rejection of established experimental tumors. Blood 100: 3155-3163, doi: 10.1182/blood-200204-1041
Knutson KL, Schiffman K, Disis ML (2001) Immunization with a HER-2/ neu helper peptide vaccine generates HER-2/neu CD8 T-cell immunity in cancer patients. J Clin Invest 107: $477-484$

Kohn DB, Sadelain M, Glorioso JC (2003) Occurrence of leukaemia following gene therapy of X-linked SCID. Nat Rev Cancer 3: 477-488, doi:10.1038/nrc1122

Lanzavecchia A (1998) Licence to kill. Nature 393: 413-414

Latouche JB, Sadelain M (2000) Induction of human cytotoxic T lymphocytes by artificial antigen-presenting cells. Nat Biotechnol 18: 405-409, doi:10.1038/74455

Maher J, Brentjens RJ, Gunset G, Riviere I, Sadelain M (2002) Human T lymphocyte cytotoxicity and proliferation directed by a single chimeric TCR/CD28 receptor. Nat Biotechnol 20: 70 -75, doi:10.1038/nbt0102-70

Morris EC, Bendle GM, Stauss HJ (2003) Prospects for immunotherapy of malignant disease. Clin Exp Immunol 131: 1-7, doi:10.1046/j.13652249.2003.02055.x

Nelson BH (2004) IL-2, regulatory T cells, and tolerance. J Immunol 172: $3983-3988$

Padua RA, Larghero J, Robin M, le Pogam C, Schlageter MH, Muszlak S, Fric J, West R, Rousselot P, Phan TH, Mudde L, Teisserenc H, Carpentier AF, Kogan S, Degos L, Pla M, Bishop JM, Stevenson F, Charron D, Chomienne C (2003) PML-RARA-targeted DNA vaccine induces protective immunity in a mouse model of leukemia. Nat Med 9: 1413 1417, doi:10.1038/nm949

Qin Z, Schwartzkopff J, Pradera F, Kammertoens T, Seliger B, Pircher H, Blankenstein $\mathrm{T}$ (2003) A critical requirement of interferon gammamediated angiostasis for tumor rejection by CD8+ T cells. Cancer Res 63: $4095-4100$

Romieu R, Baratin M, Kayibanda M, Lacabanne V, Ziol M, Guillet JG, Viguier M (1998) Passive but not active CD8+ T cell-based immunotherapy interferes with liver tumor progression in a transgenic mouse model. J Immunol 161: 5133-5137

Rooney CM, Aguilar LK, Huls MH, Brenner MK, Heslop HE (2001) Adoptive immunotherapy of EBV-associated malignancies with EBVspecific cytotoxic T-cell lines. Curr Top Microbiol Immunol 258: 221 - 229

Rosenberg SA, Yannelli JR, Yang JC, Topalian SL, Schwartzentruber DJ, Weber JS, Parkinson DR, Seipp CA, Einhorn JH, White DE (1994) Treatment of patients with metastatic melanoma with autologous tumorinfiltrating lymphocytes and interleukin 2. J Natl Cancer Inst 86: 1159 1166

Rossig C, Bollard CM, Nuchtern JG, Rooney CM, Brenner MK (2002) Epstein - Barr virus-specific human $\mathrm{T}$ lymphocytes expressing antitumor chimeric T-cell receptors: potential for improved immunotherapy. Blood 99: $2009-2016$

Sheen AJ, Sherlock DJ, Irlam J, Hawkins RE, Gilham DE (2003) T lymphocytes isolated from patients with advanced colorectal cancer are suitable for gene immunotherapy approaches. $\mathrm{Br} J$ Cancer 88: 11191127, doi:10.1038/sj.bjc.6600857

Sykulev Y, Joo M, Vturina I, Tsomides TJ, Eisen HN (1996) Evidence that a single peptide-MHC complex on a target cell can elicit a cytolytic T cell response. Immunity 4: 565-571

Van Der Bruggen P, Zhang Y, Chaux P, Stroobant V, Panichelli C, Schultz ES, Chapiro J, Van Den Eynde BJ, Brasseur F, Boon T (2002) Tumorspecific shared antigenic peptides recognized by human $\mathrm{T}$ cells. Immunol Rev 188: $51-64$

Whelan M, Whelan J, Russell N, Dalgleish A (2003) Cancer immunotherapy: an embarrassment of riches? Drug Discov Today 8: 253-258, doi:10.1016/S1359-6446(03)02633-3

Yu P, Lee Y, Liu W, Chin RK, Wang J, Wang Y, Schietinger A, Philip M, Schreiber H, Fu Y-X (2004) Priming of naïve T cells inside tumors leads to eradication of established tumors. Nat Immunol 5: 141-149, doi:10.1038/ni1029 